\title{
Effect of Polymyxin B Sulfate on Endotoxin Activity in a Gram-Negative Septicemia Model
}

\author{
JAMES J. CORRIGAN, JR., M.D., ${ }^{(18)}$ AND JAMES F. KIERNAT, B.S. \\ Department of Pediatrics, Section of Pediatric Hematology-Oncology, University of Arizona Health Sciences Center, \\ Tucson, Arizona, USA
}

\begin{abstract}
Summary
The antiendotoxin effect of polymyxin $B$ was investigated in experimentally induced septicemia in rabbits. The Pasteurella multocida organisms were sensitive to the antibacterial action of penicillin but not to polymyxin $B$. Animals pre-treated with polymyxin showed positive blood cultures and significantly reduced plasma endotoxin levels (Limulus test) with normal white blood cell and platelet counts when analyzed $6 \mathrm{hr}$ after the injection of live organisms. Polymyxin therapy given after the animals had established septicemia-endotoxemia reduced the plasma endotoxin levels and improved the survival, but had no effect on the leukopenia and thrombocytopenia. The best survival data were obtained in rabbits who were treated with both penicillin and polymyxin. The data suggest that polymyxin is effective in neutralizing the endotoxic effects from live organisms and that the timing and perhaps duration of the polymyxin treatment is of critical importance. In addition, a modified Limulus lysate method was developed which showed that quantitative plasma endotoxin determination could be made more sensitive by prior heating of the plasma to remove the natural inhibitors.
\end{abstract}

\section{Speculation}

This investigation and other reports have shown that polymyxin B sulfate neutralized endotoxin effects in animals given purified endotoxin, dead endotoxin-containing organisms, and gram-negative septicemic animals $(3,6,8,12-15)$. The antiendotoxin effect of the antibiotic appears to be separate from its antimicrobial effects. Because many of the manifestations of gram-negative septicemia in humans are reputed to be the result of endotoxemia, a clinical study focused on neutralization of the endotoxin effects would appear appropriate. The modified Limulus lysate test, as described in the report, was found to be accurate in measuring quantitative plasma endotoxin levels in small quantities of plasma by removal of the natural inhibitors by simply heating the plasma. The data also indicated that the lysate test sensitivity was directly related to the number of infectious organisms per milliliter of blood in the septicemic rabbit. Therefore, its sensitivity in humans could similarly be affected by the presence of inhibitors and the number of organisms. Because most human studies have failed to correlate the positivity of the test with the number of infecting organisms, these data would suggest that re-evaluation of its usefulness in clinical disease should be performed.

The antibiotic polymyxin B sulfate has been shown to neutralize endotoxin lethality in chick embryos and adrenalectomized mice $(8,13,15)$ and to prevent endotoxin-induced leukopenia, thrombocytopenia, disseminated intravascular coagulation, and renal cortical necrosis in rabbits $(3,14)$ and the fall in cardiac output, mean arterial pressure, and systemic vascular resistance in dogs (12). One human study suggested that endotoxin-induced changes in the neutrophils could be prevented under certain circumstances with polymyxin B sulfate (6). These data strongly suggest that polymyxin B has antiendotoxin activity which is distinct from its antibacterial power. Heretofore, all of the studies that have been reported have employed either the purified lipopolysaccharide or dead gram-negative organisms. The purpose of this study was to investigate the effect of polymyxin B on endotoxin activity in a rabbit model of gram-negative septicemia. The endotoxin effects investigated were the changes in plasma endotoxin activity, and the white blood cell and platelet counts in animals who were treated with polymyxin B sulfate.

\section{MATERIALS AND METHODS}

White rabbits of both sexes, California strain, weighing $1.0 \mathrm{~kg}$ were used. They were housed in an air-conditioned isolated animal room and fed antimicrobial free rabbit chow and water ad libitum. The production of gram-negative septicemia in these rabbits was by methods previously described (4). In essence, the animals were given $2 \times 10^{9}$ live $P$. multocida organisms per $\mathrm{kg}$ body wt ip. The animals regularly became clinically ill and had positive blood cultures and Limulus lysate tests $4-6 \mathrm{hr}$ after the injection. Sequential blood samples were obtained from the heart and were used for blood cultures, endotoxin assay and leukocyte and platelet counts. Quantitative colony counts using heparinized whole blood were performed by pour plate technique on $5 \%$ blood agar plates (Micro-Tech Diagnostics, Tucson, AZ). The plates were incubated at $37^{\circ}$ and $24 \mathrm{hr}$ later the small, mucoid gray, nonhemolytic colonies were counted.

Heparinized plasma was obtained by centrifugation of the blood at $150 \times g$ at $4^{\circ}$ for $10 \mathrm{~min}$. The plasma was divided equally and placed into two sterile plastic tubes. Each was diluted 1:10 with sterile $0.9 \% \mathrm{NaCl}$ and one was placed in a boiling water bath for $7 \frac{12}{\mathrm{~min}}$. The latter was allowed to cool and was centrifuged, and the supernatant was labeled "heated sample." Any further dilutions were made with $0.9 \%$ sterile $\mathrm{NaCl}$ solution. Limulus lysate was prepared by the method of Levin and Bang (10), and quantitative determination was based on the time for gelation to occur as determined in a photoelectric nephelometer with automatic recording device (platelet aggregometer, Chrono-Log Corp., Bromall, PA) (2, 9-11). To the cuvette was added $0.2 \mathrm{ml}$ lysate followed by $0.2 \mathrm{ml}$ test material delivered by an automatic pipette. After introduction of the test material, the time was noted on the graph paper. At the first deflection of the pen indicating a change in light transmission, the time was recorded. All samples were subsequently observed for gross gel formation. Those that did not have a definitive gel within $24 \mathrm{hr}$ were judged to have negligible or no endotoxin activity. A standard curve was constructed using known concentrations of Escherichia coli $0127 \mathrm{~B} 8$ endotoxin; the times obtained were read off the standard curve and were calculated to represent micrograms of endotoxin activity per milliliter of plasma. Each new lot of lysate was tested and was considered active if a solid gel formed between 2.5 and $4.0 \mathrm{~min}$ after the addition of $1.0 \mu \mathrm{g} \mathrm{E}$. coli endotoxin (0127:B8, Difco Laboratories, Detroit, MI, lot 503545) (5).

White blood cell counts were performed by standard hematologic technique and platelet counts were performed by phase 
microscopy employing the Unopette (Beckton, Dickinson and Co., Cockeysville, MD) (1). Polymyxin B (50 mg/vial) was obtained from Burroughs-Wellcome Co., Tuckahoe, NY. The polymyxin solutions were made up fresh daily by dissolving the antibiotic in normal saline. Antibiotic sensitivity testing of the $P$. multocida organism (kindly performed by Dr. Kenneth Ryan, Department of Pathology) showed that it was sensitive to penicillin (26-mm zone of inhibition for 10-U disc), but resistent to polymyxin B sulfate (overgrowth using 300-U disc potency) (SensiDisc, Becton, Dickinson and Co.).

All results, except the effect of treatment on mortality rate (Table 4), were statistically analyzed with use of the $t$-test for unpaired experiments. $\chi^{2}$ analysis was used for the antibiotic mortality results. A value of $P<0.05$ was considered to be significant.

\section{RESULTS}

\section{IN VIVO RABBIT EXPERIMENTS}

Sequential blood culture and unheated native plasma endotoxin activity in 39 rabbits given live $P$. multocida organisms $\left(2 \times 10^{4} / \mathrm{kg}\right.$ ip) and 20 animals given heat-killed organisms $\left(2 \times 10^{9} / \mathrm{kg}\right.$ ip $)$ are shown on Table 1. Early in the infection, the blood cultures were positive more frequently than the qualitative endotoxin assay. Eight hours after the injection, both were positive in $100 \%$ of the animals. At no time was a blood culture or lysate test positive in the control group of animals.

The relationship between the number of infecting organisms per milliliter of whole blood and endotoxin levels of unheated plasma is shown on Table 2 . As can be seen, the higher the colony forming unit, the more endotoxin that could be detected. With $10^{2}$ organisms, none had positive qualitative or quantitative lysate tests. At $10^{4}, 40 \%$ of the lysate tests were positive, yielding the

Table 1. Sequential blood culture and plasma endotoxin activity results in rabbits given live or dead organisms ip

\begin{tabular}{|c|c|c|c|c|}
\hline \multirow[b]{2}{*}{ Hour } & \multicolumn{2}{|c|}{ Live organisms (39)' } & \multicolumn{2}{|c|}{ Dead organisms $(20)^{1}$} \\
\hline & $\begin{array}{c}\text { Blood culture } \\
(\%)^{2}\end{array}$ & $\begin{array}{l}\text { Lysate test } \\
(\%)^{2}\end{array}$ & $\begin{array}{c}\text { Blood culture } \\
(\%)^{2}\end{array}$ & $\begin{array}{c}\text { Lysate test } \\
(\%)^{2}\end{array}$ \\
\hline 0 & 0 & 0 & 0 & 0 \\
\hline 2 & 82 & 33 & 0 & 0 \\
\hline 4 & 95 & 57 & 0 & 0 \\
\hline 6 & 100 & 83 & 0 & 0 \\
\hline 8 & 100 & 100 & 0 & 0 \\
\hline 12 & 100 & 100 & 0 & 0 \\
\hline 24 & & & 0 & 0 \\
\hline Death & 100 & & 0 & 0 \\
\hline
\end{tabular}

'Number of animals.

${ }^{2}$ Percent positive.

Table 2. Relationship between colony-forming units (CFU) and endotoxin levels in rabbits with positive blood cultures

\begin{tabular}{ccccc}
\hline \multicolumn{2}{c}{ Blood cultures } & & \multicolumn{2}{c}{ Plasma endotoxin } \\
\cline { 1 - 2 } \cline { 4 - 5 } $\begin{array}{c}\text { CFU } \\
\text { (bacteria/ml })\end{array}$ & $\begin{array}{c}\text { No. of obser- } \\
\text { vations }\end{array}$ & & $\begin{array}{c}\text { Native plasma } \\
(\mu \mathrm{g} / \mathrm{ml})\end{array}$ & $\begin{array}{c}\text { Heated plasma } \\
(\mu \mathrm{g} / \mathrm{ml})\end{array}$ \\
\hline $10^{2}$ & 2 & & 0.00 & 0.06 \\
$10^{4}$ & 5 & & $0.04 \pm 0.03^{1}$ & $0.08 \pm 0.06$ \\
$10^{5}$ & 11 & & $0.50 \pm 0.36$ & $4.12 \pm 2.74$ \\
$10^{6}$ & 11 & & $3.77 \pm 1.69$ & $7.81 \pm 2.49$ \\
$10^{5}$ & 6 & & $13.66 \pm 4.50$ & $37.00 \pm 9.60$ \\
$10^{8}$ & 4 & & $267 \pm 125$ & $675 \pm 275$ \\
$10^{9}$ & 6 & & $468 \pm 120$ & $1308 \pm 415$ \\
\hline
\end{tabular}

${ }^{1}$ Mean \pm SEM. mean of $0.04 \mu \mathrm{g} / \mathrm{ml}$; at $10^{3}, 64 \%$ were positive, yielding the mean of $0.50 \mu \mathrm{g} / \mathrm{ml}$; and at $10^{6}$ and above, $100 \%$ were positive with endotoxin levels ranging from $3.77-468 \mu \mathrm{g} / \mathrm{ml}$. The test plasmas were then placed in a boiling water bath as described under Materials and Methods, and subsequently tested for endotoxin activity. Comparison between the native unheated plasma and the heated sample is shown on Table 2 . As noted, the endotoxin levels ranged from $0-468 \mu \mathrm{g} / \mathrm{ml}$ in the native plasma. The heated samples all showed endotoxin activity and, when compared to its unheated mate, was always higher, generally two to three times higher. By using this technique, endotoxin activity could be detected in as few as 100 organisms per $\mathrm{ml}$ in the blood culture. To demonstrate that heating the plasma samples appeared to remove the inhibitor and did not alter the endotoxin activity of the organisms, the following experiment was done. $P$. multocida organisms, $1 \times 10^{10}$ organisms per $\mathrm{ml}$ were suspended in normal saline. The endotoxin activity in the unheated sample for this particular experiment was $6200 \mu \mathrm{g} / \mathrm{ml}$. The organisms in the saline were placed in the boiling water bath for $71 / 2 \mathrm{~min}$ and allowed to $\mathrm{cool}$, and the assay was repeated. The heated sample showed 5100 $\mu \mathrm{g} / \mathrm{ml}$ endotoxin activity. At this level of endotoxin activity, there was no significant differences between these numbers.

\section{ADMINISTRATION OF POLYMYXIN B BEFORE INJECTION OF LIVE $P$. MULTOCIDA ORGANISMS}

In these experiments, animals were given either normal saline (NSS) or polymyxin B $(5.0 \mathrm{mg} / \mathrm{kg}$ body wt) iv, $90 \mathrm{~min}$ before ip injection of $2.0 \times 10^{9}$ live $P$. multocida organisms per kg body wt. Blood samples were obtained by cardiac puncture $6 \mathrm{hr}$ after injection of the live organisms. The results of this experiment can be seen on Figure 1. Thirty-seven rabbits in whom blood samples were obtained at the start of the experiment (baseline) revealed that $0 \%$ had positive blood cultures, $0 \%$ had positive endotoxin levels, and undetectable endotoxin levels on quantitative testing existed. The white blood cell counts were 4,061 \pm 426 (mean \pm SEM) and platelet counts were $536,000 \pm 38,000$. As is noted in Figure 1, all animals who were pretreated with normal saline demonstrated positive blood cultures and lysate tests. Significant endotoxemia was noted on quantitative analysis with significant leukopenia and thrombocytopenia also present at this time. Eighty percent $(80 \%)$ of the polymyxin-treated animals had positive blood cultures; the endotoxin activity, although present, was significantly less $(P<0.001)$ than in the saline-treated controls. Animals given polymyxin B showed no leukopenia or thrombocytopenia with 5.0 $\mathrm{mg} / \mathrm{kg}$ body wt dosage.

\section{POLYMYXIN ADMINISTERED 6 HR AFTER THE INJECTION OF LIVE P. MULTOCIDA ORGANISMS}

In this study, the antibiotic was given iv $6 \mathrm{hr}$ after the ip injection of the live organisms $\left(2 \times 10^{9} / \mathrm{kg}\right)$. Cardiac samples were then obtained $0.5-3 \mathrm{hr}$ after the administration of the antibiotic. The results of this study are shown in Figure 2 . The blood cultures remained positive in $100 \%$ of the animals at both time periods. Endotoxin activity was reduced at both time periods. However, by the $3 \mathrm{rd} \mathrm{hr}$, the endotoxin activity had increased in those animals given polymyxin but was still lower than the saline-treated controls. The animals were leukopenic and thrombocytopenic at the time of the injection of the polymyxin and remained so throughout the testing period. Four of the saline and nine of the polymyxin-treated rabbits died after cardiac puncture at $6 \frac{1 / 2}{\mathrm{hr}}$.

\section{PENICILLIN EXPERIMENTS}

Rabbits were infected with the ip injection of live organisms and at $6 \mathrm{hr}$ were given the intravenous injection of $10,000 \mathrm{U} / \mathrm{kg}$ body wt aqueous penicillin. Blood samples were obtained $10 \mathrm{~min}$ after the injection of the antibiotic. Results of these experiments are shown in Table 3. As can be seen, the blood cultures were positive before treatment and negative after the penicillin. There 
was no significant change in the endotoxin levels, white blood cell counts, or platelet counts between these two groups of animals.

\section{MORTALITY STUDY}

Animals were given the live $P$. multocida organism ip and then at $6 \mathrm{hr}$ a single iv injection of either saline, penicillin, polymyxin, or penicillin plus polymyxin. Twelve and $24-\mathrm{hr}$ mortality was noted and the results are shown in Table 4. As can be seen, the lowest mortality was in the penicillin plus polymyxin treatment group. Compared to saline-treated animals, mortality was signifi-
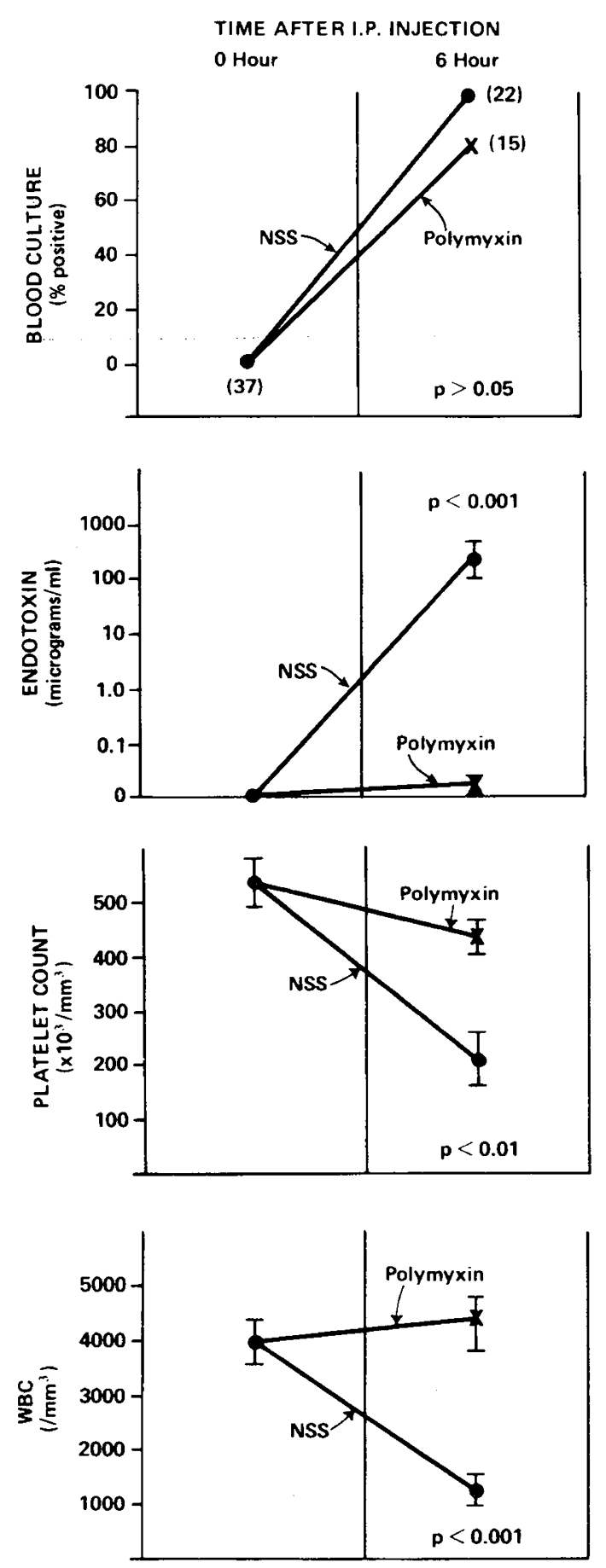

Mean \pm SEM

$(1)=$ Number of Animals

Fig. 1. Rabbits pretreated with polymyxin B before ip injection of $P$. multocida. Effect on endotoxin levels and white blood cell and platelet counts in gram-negative septicemia.
TIME AFTER I.P. INJECTION
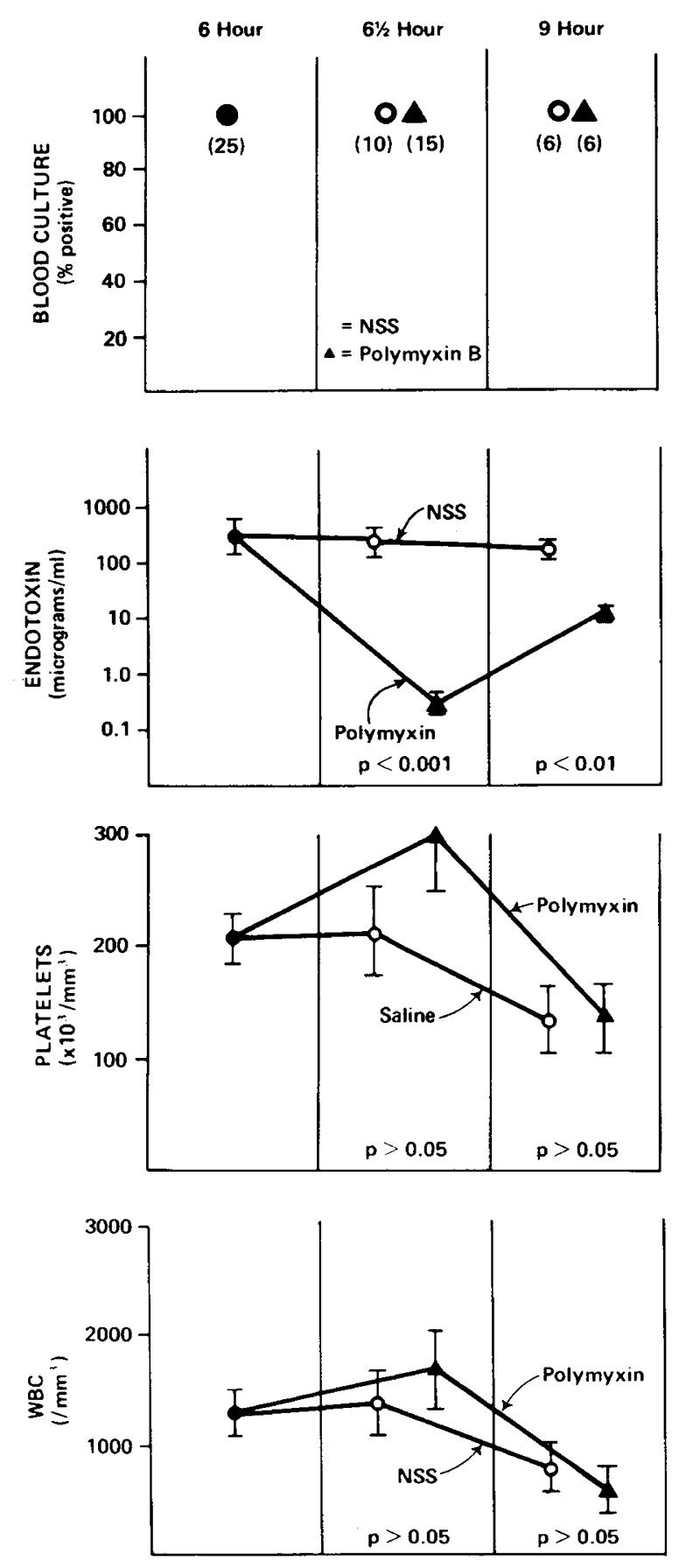

Mean : SEM

$(1)=$ Number of Animals

Fig. 2. Effect of polymyxin B administered after septicemia has been established (antibiotic given $6 \mathrm{hr}$ after ip injection of $P$. multocida).

cantly less for penicillin $(P<0.001)$ and penicillin plus polymyxin $(P<0.001)$-treated rabbits at both 12 and $24 \mathrm{hr}$. Although the mortality in the penicillin plus polymyxin-treated animals was lower, it was not significantly different from the penicillin-treated group at 12 or $24 \mathrm{hr}$.

\section{DISCUSSION}

Previous studies have shown that endotoxin-induced leukopenia, thrombocytopenia, disseminated intravascular coagulation, Shwartzman reactions, shock, and death can be abolished by polymyxin B sulfate $(3,6,8,12-15)$. The mechanism by which the 
Table 3. Effect of penicillin ${ }^{1}$ treatment on plasma endotoxin levels

\begin{tabular}{lcccc}
\hline & $\begin{array}{c}\text { Blood culture } \\
(\% \text { positive })\end{array}$ & $\begin{array}{c}\text { Endotoxin } \\
(\mu \mathrm{g} / \mathrm{ml})\end{array}$ & WBC $\left(/ \mathrm{mm}^{3}\right)$ & $\begin{array}{c}\text { Platelets } \\
\left(\times 10^{3} / \mathrm{mm}^{3}\right)\end{array}$ \\
\hline $\begin{array}{c}\text { Pre-penicillin } \\
(5)^{2}\end{array}$ & 100 & $124 \pm 68^{3.3}$ & $1166 \pm 273$ & $195 \pm 39$ \\
$\begin{array}{c}\text { Post-penicillin } \\
(5)^{2}\end{array}$ & 0 & $146 \pm 98$ & $2090 \pm 495$ & $135 \pm 35$
\end{tabular}

$(5)^{2}$

'Penicillin given $6 \mathrm{hr}$ after injection of live organisms.

2 Number of animals.

${ }^{3}$ Mean \pm SEM.

Table 4. Mortality in rabbits with gram-negative septicemia

\begin{tabular}{lcc}
\hline & \multicolumn{2}{c}{ Mortality (\%) } \\
\cline { 2 - 3 } \multicolumn{1}{c}{ Treatment $^{1}$} & $12 \mathrm{hr}$ & $24 \mathrm{hr}$ \\
\hline Saline (75) & 69 & 100 \\
Penicillin G (33) & 33 & 70 \\
Polymyxin B (14) & 64 & 86 \\
Penicillin + polymyxin (14) & 21 & 50
\end{tabular}

${ }^{1}$ Animals were given saline, penicillin $\mathrm{G}(10,000 \mathrm{U} / \mathrm{kg})$, polymyxin $\mathrm{B}$ $(5 \mathrm{mg} / \mathrm{kg})$, or penicillin + polymyxin as one injection $6 \mathrm{hr}$ after inoculation.

${ }^{2}$ Number in parentheses represents number of animals.

antibiotic neutralizes endotoxin toxicity appears to je that of a cationic detergent (3). In the present study, endotoxin effects (thrombocytopenia, leukopenia, and positive Limulus lysate tests) occurring during the course of experimentally induced gram-negative septicemia could similarly be abolished with polymyxin B. Because the microorganisms employed in these experiments were not sensitive to the antibacterial power of the antibiotic, the changes were due to the ability of polymyxin to detoxify the endotoxin. The rabbits in the study were either pretreated with polymyxinor given the antibiotic after the establishment of the septicemic state. The purpose of pretreating the animals was 2fold. First, it might be argued that the reduced endotoxin activity as measured by the Limulus lysate test could be artifactual inasmuch as both organisms and polymyxin were in the blood sample that was tested so that the neutralization might have occurred in vitro rather than in vivo. However, the reduced endotoxin activity corresponded with the lack of demonstration of other known endotoxic events at the same time. Thus, the classical demonstration of leukopenia and thrombocytopenia was not observed in the pretreatment animals at a time when the blood cultures were positive. Thus, our interpretation is that the reduced endotoxin activity was indeed a measure of in vivo endotoxin levels. The second reason had to do with the time of administration of the antibiotic. It has been shown by us and other investigators that the timing of the polymyxin injection appears to be critical in order to effectively neutralize the endotoxin $(3,6,7,14)$. In the pretreatment animals, the polymyxin was given before the establishment of an endotoxemic state and, as can be seen by the data, effectively prevented the development of endotoxin-induced leukopenia and thrombocytopenia. However, in the experiments in which the polymyxin was given after the establishment of the septicemic-endotoxemic state, there was no observable change in the leukopenic-thrombocytopenic state, even though the plasma endotoxin activity was noted to be significantly reduced. Thus, the data from these experiments using the live organisms corroborate the findings in animals given lipopolysaccharide and show that, once the endotoxin has exerted its effect at the tissue level, the toxin reaction cannot be neutralized by the polymyxin $\mathrm{B}(3,7)$. Because bacterial endotoxins are reputed to be responsible for some of the clinical manifestations of human diseases caused by gram-negative organisms, and because the endotoxin toxicity, elicited either by purified lipopolysaccharide, dead gram-negative organisms, or the septicemic state, can be neutralized by the antibiotic, it would appear reasonable to suggest that a clinical investigation in human disease is warranted.

As part of this investigation, a modification of the Limulus lysate test for detecting plasma endotoxin levels was developed. Levin et al. in 1970 reported the use of the lysate test for detecting endotoxin human blood and, in addition, demonstrated the presence of a natural plasma inhibitor (11). The prevailing method for separating the endotoxin from plasma inhibitors utilizes a chloroform extraction. However, the volumes needed to perform this extraction have precluded this method for standard use in small pediatric patients and small animals. The data in the current study showed that the positivity of the lysate test and the quantitative amount of endotoxin measured were related to the number of microorganisms per milliliter of whole blood and to the natural inhibitor levels of plasma. The quantitative plasma endotoxin levels could be made more sensitive by prior heating of the plasma which removed the natural inhibitors. By using this modification, as little as $10^{2}$ organisms were found to give a positive test in the rabbit model. Thus, these data indicate that the Limulus lysate test can be used to detect and quantitate endotoxin activity in small samples of plasma and should have application in pediatric practice.

\section{REFERENCES AND NOTES}

I. Brecher, G., and Cronkite, E. P.: Estimation of the number of platelets by phase microscopy. In: L. M. Tocantins, L. A. Kazal: Blood Coagulation, Hemorrhage and Thrombosis, p. 52 (New York, Grune \& Stratton, Inc., 1964).

2. Cooper, J. F., Levin, J., and Wagner, H. N., Jr.: Quantitative comparison of in vitro and in vivo methods for the detection of endotoxin. J. Lab. Clin. Med., 78: 138 (1971)

3. Corrigan, J. J., Jr., and Bell, B. M.: Endotoxin induced intravascular coagulation: Prevention with polymyxin B sulfate. J. Lab. Clin. Med., 77: 802 (1971).

4. Corrigan, J. J.. Jr., and Kiernat, J. F.: Effect of heparin in experimental gramnegative septicemia. J. Infect. Dis., /31: 138 (1975).

5. Corrigan, J. J., J⿸厂 J., and Kiernat. J. F.: Experimental septicemia: Evaluation of lysate test for endotoxin. Pediat. Res., 10: 397 (1976).

6. Corrigan, J. J., Jr., Seiber. O. F., Jr., Ratajczak, H., and Bennett, B. B.: Modification of human neutrophil response to endotoxin with polymyxin B sulfate. J. Infect. Dis.. 130: 384 (1974).

7. Craig. W. A., Turner, J. H., and Kunin. C. M.: Prevention of the generalized Shwartzman reaction and endotoxin lethality by polymyxin B localized in tissues. Infect. Immun.. 10: 287 (1974).

8. Gruninger, R. P., and Howe. C. W.: Temporal protection of chick embryo against endotoxin by isoproterenol. polymyxin B sulfate and hydrocortisone. Proc. Soc. Exp. Biol. Med., 130: 1202 (1969).

9. Jorgensen. J. H., Chipps, C. B. E., and Smith. R. F.: Rapid detection of gramnegative bacteriuria by use of the Limulus endotoxin assay. Appl. Microbiol.. 26: 38 (1973).

10. Levin, J., and Bang. F. B.: Clottable protein in Limulus: Its localization and kinetics of its coagulation by endotoxin. Thromb. Diath. Haemorrh.. 19: 186 (1968).

11. Levin, J.. Tomasulo. P. A.. and Oser. R. S.: Detection of endotoxin in human blood and demonstration of an inhibitor. J. Lab. Clin. Med.. 75: 903 (1970).

12. Palmer. J. D. and Rifkind. D.: Neutralization of the hemodynamic effects of endotoxin by polymyxin B. Surg. Gynecol. Obstet.. 138: 755 (1976).

13. Rifkind. D.: Prevention by polymyxin B of endotoxin lethality in mice. $J$. Bacteriol., 93: 1463 (1967).

14. Rifkind, D.. and Hill. R. B.. Jr.: Neutralization of the Shwartzman reactions by polymyxin B. J. Immunol.. 99: 564 (1967).

15. Rifkind. D.. and Palmer. J. D.: Neutralization of endotoxin toxicity in chick embryos by antibiotics. J. Bacteriol.. 92: 815 (1966).

16. This research was supported by Research Grant AI 10353 from the National Institute of Allergy and Infectious Diseases from the National Institutes of Health. Bethesda. MD.

17. This paper was presented in part at the 87th Annual Meeting of the American Pediatric Society and the Society for Pediatric Research, San Francisco, CA, April 1977.

18. Requests for reprints should be addressed to: Dr. Corrigan, University of Arizona Health Sciences Center, Tucson, AR 85724 (USA).

19. Received for publication October 31, 1977.

20. Accepted for publication March 7, 1978. 